\title{
LA 12'-HYDROXYISOSTRYCHNOBILINE: NOUVEL ALCALOÏDE DU STRYCHNOS VARIABILIS
}

\section{Monique Tits*, Dirk Tavernier† et Luc Angenot*}

Laboratoire de Pharmacognosie, Institut de Pharmacie de l’Université de Liège, Rue Fusch, 5, B-4000 Liège, Belgium; †Department of Organic Chemistry, NMR Spectroscopic Unit, Rijksuniversiteit Gent, Krijgslaan, 271 (S 4 bis) B-9000 Gent, Belgium

(Reçu le 22 août 1978) Key Word Index-Strychnos variabilis; Loganiaceae; bisindole alkaloids; isostrychnobiline; 12 '-hydroxyiso-
strychnobiline.

Abstract - The structure of a new bisindole alkaloid, 12'-hydroxyisostrychnobiline, has been proposed from the analysis of its $300 \mathrm{MHz}^{1} \mathrm{H}$ NMR spectrum and comparison of the spectroscopic data with those of various monomeric and dimeric alkaloids, previously isolated from the same Strychnos species

\section{INTRODUCTION}

Dans une publication antérieure, nous avons signalé quelesalcaloïdes dimères dihydroindoliquesasymétriques traient largement majoritaires dans l'écorce des racines 5. Strychnos variabilis. Deux d'entre-eux, la strychnoaline et l'isostrychnobiline (formule brute $\mathrm{C}_{40} \mathrm{H}_{46} \mathrm{~N}_{4} \mathrm{O}_{2}$ -PM 614) ont déjà fait l'objet d'une étude ayant mis en Evidence leur originalité [1].

Un troisième dimère asymétrique dihydroindolique de formule brute $\mathrm{C}_{40} \mathrm{H}_{46} \mathrm{~N}_{4} \mathrm{O}_{3}$ - PM 630, déterminée par spectrométrie de masse à haute résolution vient ègalement fètre isolé. Son identification structurale et l'établisse-

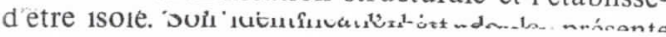
ment de sa stéréochimie font l'objet de la présente communication. La stéréochimie de l'isostrychnobiline, jusqu’à présent inconnue, est également précisée.

\section{RESULTATS ET DISCUSSION}

L'analyse des différents spectres (UV, masse, IR, ${ }^{1} \mathrm{H}$ RMN) permet de constater une relation étroite entre le nouvel alcaloïde et l'isostrychnobiline [1]. Des ressemblances particulièrement frappantes existent au niveau des spectres de ${ }^{1} \mathrm{H}$-RMNà champ élevé $(300 \mathrm{MHz})$. On y distingue notamment les éléments structuraux suivants: 2 chaînes éthylidéniques (dont un des doublets attribué au $\mathrm{Me}_{18}$ est anormalement blindé); 1 fonction $N$-acétyle; 1 fonction carbinolamineéther.

Dans le case du nouvel alcaloïde, on notera cependant la présence d'un singulet à $9.97 \mathrm{ppm}$ compatible avec une fonction phénolique située d'après l'analyse des protons aromatiques en position 9 ou 12 (ou bien $9^{\prime}$ ou $12^{\prime}$ ). Cette fonction phénolique est également détectée dans le spectre de masse par la présence d'un fragment à $m / e 160$. Par contre, le spectre UV ne subit pas de modification en milieu alcalin, ce qui révèle l'existence d'une fonction cryptophénolique du même type que celle existant dans le cas de la vomicine, c'est-à-dire provenant d'une liaison hydrogène entre le $\mathrm{N}$-acétyle et l'OH phénolique qui dès lors ne peut se trouver qu'en position $12^{\prime}$.

C'est en comparant les spectres ${ }^{1} \mathrm{H}$ RMN $(300 \mathrm{MHz})$ de ces deux alcaloïdes avec ceux des bases monomères: la rétuline $(16 \beta \mathrm{H})$, l'isorétuline $(16 \alpha \mathrm{H})$ et leurs dérivés $N$-désacétylés dont l'étude détaillée est décrite ailleurs [2], que l'on a pu préciser leur stéréochimie qui est identique dans les deux cas. La rotation empêchée autour de la liaison amide ( $N$-acétyle) donne lieu en principe à deux rota-

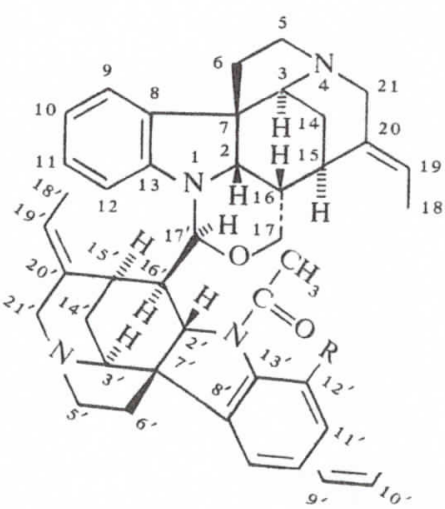

Isostrychnobiline: $\mathrm{R}=\mathrm{H}$

12'-Hydroxyisostrychnobiline: $\mathrm{R}=\mathrm{OH}$

mères. Dans la 12'-hydroxyisostrychnobiline, la liaison hydrogène entre la fonction phénolique et l'oxygène de l'acétyle stabilise un de ces rotamères à l'exclusion de l'autre. L'isostrychnobiline, quant à elle, présente les deux rotamères dans des proportions $30-70 \%$, rendant le spectre RMN beaucoup plus complexe. Néanmoins, la plupart des atomes d'hydrogène peuvent être repérés dans ces spectres, grâce aux expériences de découplage et d'INDOR. Leurs constantes de couplage figurent dans la partie expérimentale.

La 12'-hydroxyisostrychnobiline et l'isostrychnobiline sont toutes les deux composées d'une partie rétuline' et d'une 'partie isorétuline'. Cette conclusion découle immédiatement des différences au niveau des couplages à longue distance entre les protons $\mathrm{H}_{21}-\mathrm{H}_{19}-\mathrm{Me}_{18}$. Ainsi, le signal de $\mathrm{Me}_{18}$ provenant de la partie isorétuline se présente comme un quadruplet bien défini, tandis que la partie rétuline donne lieu à un doublet élargi pour le groupe méthyle [2]. Les constantes de couplage $J_{\mathrm{H} 2-\mathrm{H} 16}$ $=8.7 \mathrm{~Hz}$ et $J_{\mathrm{H}^{\prime} \text {-H16 }}=10.2 \mathrm{~Hz}$ permettent d'attribuer à $\mathrm{C}_{16}$ la configuration $16 \beta \mathrm{H}$ (partie rétuline) et à $\mathrm{C}_{16}$, la configuration $16^{\prime} \alpha \mathrm{H}$ (partie isorétuline) [3, 4]. La configuration du $\mathrm{C}_{17}$, doit maintenant être déterminée.

On doit attribuer une forme bateau au cycle oxazine pour pouvoir expliquer le déplacement du $\mathrm{Me}_{18}$ dans le spectre ${ }^{1} \mathrm{H}$ RMN vers les champs élevés (1.44 ppm pour la $12^{\prime}$-hydroxyisostrychnobiline, 1.39 et $1.56 \mathrm{ppm}$ pour 
les deux rotamères de l'isostrychnobiline). En effet, lors de la construction du stéréomodèle moléculaire, nous voyons que si le cycle oxazine a une forme bateau, ce méthyle se trouve dans la région blindante du champ du noyau aromatique de l'autre monomère. Dès lors, le $\mathrm{H}_{17}$, est en $\alpha$ et la configuration de tous les atomes de carbone asymétriques est établie.

Dans le cas de l'isostrychnobiline, il est intéressant de noter que la constante de couplage $J_{\mathrm{H} 16^{\prime}-\mathrm{H}_{17}}$, est égale à $9.5 \mathrm{~Hz}$ pour le rotamère majoritaire et égale à $6 \mathrm{~Hz}$ pour le rotamère minoritaire. La rotation du groupe amide est donc liée à une autre rotation (partielle) autour de la liaison $\mathrm{C}_{16},-\mathrm{C}_{17}$.

En conclusion, l'identification de la 12'-hydroxyisostrychnobiline a été facilitée grâce aux relations que l'on a pu établir entre ses spectres et ceux des autres dimères. La simplification de son spectre ${ }^{1} \mathrm{H}$ RMN (300 MHz) a permis une étude complète de sa stéréochimie et par conséquent de celle de l'isostrychnobiline, son homologue non phénolique. L'étude de la stéréochimie de la strychnobiline est en cours.

\section{PARTIE EXPERIMENTALE}

Matériel. Le matériel étudié a déjà été décrit précédemment [1]. Il est constitué d'écorces de racines de Strychnos variabilis. Extraction et fractionnement. Les méthodes d'extraction et de purification sont aussi les mèmes que celles utilisées pour la strychnobiline et l'isostrychnobiline [1]

$12^{\prime}$-Hydroxyisostrychnobiline. Poudre blanche, très amère $R$ dans la phase: EtOAc, iso- $\mathrm{PrOH}, \mathrm{NH}, \mathrm{OH}(45: 35: 5)=0.75$ Coloration violette avec le sulfate cérique. UV $\lambda_{\max }^{\mathrm{MeOH}} \mathrm{nm}(\log \varepsilon)$ : 211.5 (4.64), 250 (4.17), 290 (3.7), épaulement a 308 (3.43) 3pectres identiques en milieu acide et alcalin. $\mathrm{DC}[\Theta]=+52470$ $(253 \mathrm{~nm}) . \quad$ IR $\tilde{v}_{\max }^{\mathrm{KBr}} \mathrm{cm}^{-1}: 2930(\mathrm{C}-\mathrm{H}), 1630$ (amide), 1605 $(\mathrm{C}=\mathrm{C}), 1480,780$ (benzène trisubstitué), 755, 742 (benzène ortho disubstitué), SM $220^{\circ} 70 \mathrm{eV} \mathrm{m} / \mathrm{e}$ (abondance en pourcentage du pic de base): $630(11),\left[\mathrm{M}^{+} \mathrm{C}_{40} \mathrm{H}_{46} \mathrm{~N}_{4} \mathrm{O}_{3}-\right.$ mes. 630.3579 , - calc. 630.3567], 602(3), 601(7), 587(2), 480(3), 479(5), 466(3), 353(6), 346(2), 337(5), 334(4), 333(2), 321(5), 316(5), 315(6), $310(4), 309(6), 308(50), 307(100 \%), 279(8), 249(5), 172(8), 160(14)$, $156(6), 149(8), 144(20), 136(7), 134(10), 122(7), 121(16), 119(8)$, 108(11), $106(11) .{ }^{1} \mathrm{H}-\mathrm{RMN}\left(\mathrm{CDCl}_{3}\right) 300 \mathrm{MHz}: \delta 9.97(\mathrm{OH}$ phénolique sur $\mathrm{C}_{12}$. disparaît avec $\left.\mathrm{D}_{2} \mathrm{O}\right), 7.18\left(t, \mathrm{H}_{10}\right), 6.92$ $\left(d, \mathrm{H}_{11}\right), 6.70\left(d, \mathrm{H}_{9}\right), 7.16\left(t, \mathrm{H}_{11}\right), 7.11\left(d, \mathrm{H}_{9}\right), 6.81\left(t, \mathrm{H}_{10}\right)$, $6.57\left(d, \mathrm{H}_{12}\right), 5.65\left(q, \mathrm{H}_{19^{\prime}}\right), 5.36\left(q, \mathrm{H}_{19}\right), 5.11\left(d, \mathrm{H}_{1}, J_{17}+16^{\prime}=\right.$
$9.2 \mathrm{~Hz}), 4.22\left(d, \mathrm{H}_{2}, J_{2} \cdot 16^{\prime}=10.2 \mathrm{~Hz}\right), 3.82\left(d, \mathrm{H}_{2} J_{2} 16=8.75\right.$ $\mathrm{Hz}), 3.72\left(\mathrm{H}_{3}, W_{\frac{1}{2}}=9 \mathrm{~Hz}\right), 3.67\left(\mathrm{H}_{21} \cdot J_{21^{\prime} \mathrm{A} 21^{\prime} \mathrm{B}}=14 \mathrm{~Hz}\right), 3.64$ $\left(\mathrm{H}_{21 \mathrm{~A}} J_{21 \mathrm{~A} 21 \mathrm{~B}}=14 \mathrm{~Hz}\right), 3.54\left(\mathrm{H}_{3} W_{1}=8 \mathrm{~Hz}\right), 3.38\left(\mathrm{H}_{17 \mathrm{~A}}\right.$
$\left.{ }^{2} J_{17 \mathrm{~A} 17 \mathrm{~B}}=11.5 \mathrm{~Hz} J_{17 \mathrm{~A} 16}=4 \mathrm{~Hz}\right), 3.36\left(\mathrm{H}_{15^{\prime}} J_{16^{\prime} 15^{\circ}}=3.5\right.$ $\mathrm{Hz}), 3.25\left(\mathrm{H}_{17 \mathrm{~B}} J_{17 \mathrm{~B} 16}=11 \mathrm{~Hz}\right), 3.21\left(\mathrm{H}_{21^{\prime} \mathrm{B}} J_{21^{\prime} \mathrm{B} \mathrm{Me} 18^{\prime}}=2 \mathrm{~Hz}\right.$ : couplage homoallylique $J_{21^{\prime} \text { B19' }}=2 \mathrm{~Hz}$ : couplage allylique), $2.97\left(\mathrm{H}_{21 \mathrm{~B}}\right), 2.39\left(\mathrm{H}_{15}\right), 2.26\left(\mathrm{H}_{16}\right), 2.09\left(\mathrm{~m}, \mathrm{H}_{16}\right), 2.09(\mathrm{~s}, 3 \mathrm{H}$, $\left.-\mathrm{N}-\mathrm{C}-\mathrm{CH}_{3}\right), 1.69\left(d d, 3 \mathrm{H}, \mathrm{Me}_{18}\right), 1.44\left(d, 3 \mathrm{H}, \mathrm{Me}_{18}\right)$. II $\mathrm{O}$

Isostrychnobiline. Les spectres UV, IR, SM, ${ }^{1} \mathrm{H}$ RMN $(90 \mathrm{MHz})$ ont été décrits antérieurement [1]. Le rotamère a est celui où le carbonyle est dirigé vers le cycle benzénique. Rotamere $\mathrm{a} \simeq 70 \%$, rotamère $\mathrm{b} \simeq 30 \% \cdot{ }^{1} \mathrm{H}-\mathrm{RMN}\left(\mathrm{CDCl}_{3}\right) 300 \mathrm{MHz} 0^{\circ}$ $\delta 7.84\left(d, \simeq 0.7 \mathrm{H}, \mathrm{H}_{12}\right.$, rotamère a), entre 7.30 et 7.04 (massif de protons aromatiques), $6.79\left(t, \mathrm{H}_{11}\right), 6.66\left(t, \mathrm{H}_{10}\right), 6.54\left(d, \mathrm{H}_{9}\right)$, $6.33\left(d, \simeq 0.3 \mathrm{H}, \mathrm{H}_{12}\right.$, rotamère $\left.\mathrm{b}\right), 5.66\left(q, \mathrm{H}_{19}\right.$, rotamère $\left.\mathrm{a}\right)$, $5.57\left(q, \mathrm{H}_{19}\right.$, rotamère b), $5.38\left(q, \mathrm{H}_{19}\right.$ rotamères a et b), 5.27 $\left(d, \mathrm{H}_{17^{\prime}}, J_{17^{\prime} 16^{\prime}}=6 \mathrm{~Hz}\right.$ rotamère b), $5.14\left(d, \mathrm{H}_{2}, J_{2,16^{\prime}} \simeq 10 \mathrm{~Hz}\right.$ rotamère $\mathrm{b}), 5.12\left(d, \mathrm{H}_{17^{\prime}}, J_{17^{\prime} 16^{\circ}}=9.5 \mathrm{~Hz}\right.$ rotamère a), 4.22 $\left(d, \mathrm{H}_{2}, J_{2}{ }^{16^{\prime}}=10.6 \mathrm{~Hz}\right.$ rotamère a), $3.90\left(d, \mathrm{H}_{2} J_{216}=8.7 \mathrm{~Hz}\right.$ rotamère b), $3.71\left(d, \mathrm{H}_{2} J_{216}=8.7 \mathrm{~Hz}\right.$ rotamère a), 3.37 $\left(\mathrm{H}_{15}\right), 3.37\left(\mathrm{H}_{17 \mathrm{~A}} J_{17 \mathrm{~A} 16}=4 \mathrm{~Hz} J_{17 \mathrm{~A} 17 \mathrm{~B}}=11 \mathrm{~Hz}\right), 3.24\left(\mathrm{H}_{17 \mathrm{~B}}\right.$ $\left.J_{17 \mathrm{~B} 16}=11 \mathrm{~Hz}\right), 2.36\left(\mathrm{H}_{15}\right), 2.27\left(\mathrm{H}_{16}\right), 2.10\left(\mathrm{H}_{16}\right.$ rotamère $\left.\mathrm{a}\right)$, $2.03 \mathrm{H}_{16}$. rotamère $\left.\mathrm{b}\right), 2\left(s, 3 \mathrm{H}, \mathrm{N}-\mathrm{C}-\mathrm{CH}_{3}\right), 1.71(d d, \simeq 2.1 \mathrm{H}$, $\mathrm{O}$

$\mathrm{Me}_{18}$, rotamère a), $1.67\left(d d, \simeq 0.9 \mathrm{H}, \mathrm{Me}_{18}\right.$, rotamère $\left.\mathrm{b}\right), 1.56$ $\left(d, 1 \mathrm{H}, \mathrm{Me}_{18}\right.$ rotamère b), $1.39\left(d, 2 \mathrm{H}, \mathrm{Me}_{18}\right.$ rotamère a).

Remerciements-Nous tenons à exprimer nos plus vifs remerciements à Monsieur le Professeur G. Van Binst (Vrije Universiteit te Brussel), aui s'est chargé de la prise des spectres de masse à haute résolution.

\section{BIBLIOGRAPHIE}

1. Tits, M. et Angenot, L. (1978) Planta Med. 34, 60

2. Tavernier, D., Anteunis, M., Tits, M. et Angenot, L. (1978) Bull. Soc. Chim. Belg. 87, 595.

3. Wenkert, E. et Sklar, R. (1966) J. Org. Chem. 31, 2689.

4. Hymon, J. R. et Schmid, H. (1966) Helv. Chim. Acta 49. 2067. 